Journal of Social Sciences 5(4): 319-328, 2009

ISSN 1549-3652

(C) 2009 Science Publications

\title{
Relationship between Affordances in the Home Environment and Motor Development in Children Age 18-42 Months
}

\author{
Alireza Haydari, Parviz Askari and Maryam Zarra Nezhad \\ Department of Psychology, Faculty of Humanities Sciences, \\ Azad University of Ahvaz, Ahvaz, Iran
}

\begin{abstract}
Problem statement: Environmental stimulation plays a critical role in optimal human development during the early stages of life and an optimal level (of development) occurs with strong contextual support. It is well accepted among develop mentalists that motor-perceptual-and cognitive development are fundamentally interrelated. This suggests that status of motor development is an important factor in overall child well-being. The objectives of this study were to investigate (i) the validity and reliability of AHEMD-SR in Iran and (ii) the relationship between affordances and level of motor development. The instrument could provide useful information in a wide variety of settings, including applications to intervention and remediation. Approach: Data were collected throughout random sampling from 350 families with children within the age range 18-42 months in early childhood centers in Ahvaz, Iran. Families were asked to complete an inventory containing 67 items (AHEMD-SR) which represent five factors: Outside physical space, inside physical space, variety of stimulation, gross motor materials and fine motor materials. Validity, reliability, correlations and regression were examined. Results: The findings of the research suggested that the validity test of AHEMD-SR equals 0.75 and its reliability test is 0.89 . A significant $(0.01$ or $0.05)$ positive moderate correlation was found between the five factors of AHEMD and Total AHEMD. Furthermore, there was a multiple relationship between the five factors of AHEMD and total AHEMD. Conclusion/Recommendations: For the first time in Iran it has been showed that AHEMDSR is a valid (0.75) and reliable (0.89) instrument for assessing how well environments home afford movement and potentially promotes motor development. A significant $(0.01$ or 0.05$)$ positive moderate correlation was found between the five factors of AHEMD and Total AHEMD. There are also strong and significant multi relationships between dependent variable and predictors (the five factors). The best predictor of Total AHEMD is fine motor toys.
\end{abstract}

Key words: Early childhood, affordances, motor skills, gross and fine motor materials, variety of stimulation

\section{INTRODUCTION}

Because the human nervous system is immature at the time of birth, children are expected to grow and develop continually throughout their childhood years. A number of factors combine to influence each child's rate and quality of motor development. The first years of life are the most important stage in human being development. Recent researches in child development indicate that an optimal range of development occurs with a stimulating environment and strong contextual support $^{[1,2]}$. New motor skills must be practiced, or rehearsed, in order to become strong, fluid and well coordinated. Given enough practice, some movements become so automatic that we are hardly aware of them and need not attend to how well we are executing them. For most children, the development of motor skills occurs throughout childhood and follows certain predictable steps or stages, also known as developmental milestones ${ }^{[3]}$.

Of the various factors comprising the environment, few would disagree that the home (representing the family) is a primary agent for learning and development. For the past 40 years, effort has been devoted to mapping the relations between the home environment and selected aspects of the child's development. For most children, interior of the home and its immediate surroundings are the first environments they experience throughout their early years. Young children, spend the majority of their time

Corresponding Author: Alireza Haydari, Department of Psychology, Faculty of Humanities Sciences, Azad University of Ahvaz, Ahvaz, Iran 
in the home. Home environments have been shown to be a major factor that influences the overall development of children. Availability of stimulating objects, books and play materials within the home are critical indicators for the overall quality of the home environment. Early motor development has long been used by healthcare providers and parents as an indicator of progress of neurological development during infancy $^{[4]}$ and it is a particular focus of monitoring infants born preterm ${ }^{[5]}$.

Current theories of early motor development suggest that the acquisition of new motor abilities arises from the interaction of multiple elements of the infant, the environment and the task at hand through a process of exploration of movement options and selection of the optimal solution in a given context ${ }^{[6]}$. Each aspect of development is intricately linked and if one aspect is hampered or neglected in some way a child will fail to reach her full potential. It is the responsibility of the child's careers to ensure that all needs are met.

The level of skill that children display in performing certain activities increases as they develop. As children develop so does their play. Play is as important for a child's developmental needs as good nutrition, warmth and protection. It provides opportunities to improve gross and fine motor skills and maintain physical health. It helps to develop imagination and creativity, provides a context in which to practice social skills, acts as an outlet for emotional expression and provides opportunities to understand value systems. Providing for play includes ensuring that the child has opportunities, resources and time for play appropriate to her stage of development.

The ecological environment is defined by Bronfenbrenner and $\mathrm{Ceci}^{[7]}$ as a set of "nested structures" composed of microsystems, mesosystems, exosystems, macrosystems and chronosystems. The microsystem is "a pattern of activities, roles and interpersonal relations experienced by the developing person in a given setting with particular physical and material characteristics ${ }^{[8]}$," The home environment, including the parent-child relationship, is an example of a microsystem. A mesosystem includes the interrelations among two or more microsystems in which the developing child participates (for example, the relations between parents and extended family members and friends). The exosystem refers to processes between or among two or more settings, only one of which contains the developing child (for example, relations between children and their mothers and between mothers and the gambling casino). The macrosystem includes influences of the broader cultural or socioeconomic environments, such as the 1988
Indian Gaming and Regulatory Act (IGRA) and employment opportunities in the larger social environment that are available to families. The chronosystem accounts for the influence on the child's development of consistency and change over the life course, such as consistency and stability of developmentally appropriate parenting behaviors and activities. These ecological structures serve as an overarching framework for this study.

The starting premise, founded in selected propositions of ecological theory of Gibson ${ }^{[9,10]}$ underscores the notion that the home provides affordances that can be conducive to stimulating motor development. Although the term affordance has been interpreted in several ways, ours is one of a more critical nature. Affordances are opportunities that offer the individual potential for action and consequently to learn and develop a skill or a part of the biological system $^{[11]}$. In addition to the more obvious set of affordances such as toys, materials, apparatus and availability of space, stimulation and nurturing by parents (and others) provide the additional component of events. This view agrees with Stoffregen ${ }^{[12]}$ and Hirose $^{[13]}$ in that events can be affordances-events offer the child opportunities for action. Hirose stated, "Affordances are opportunities for action that objects, events, or places in the environment provide for the animal".

Play materials and toys are obvious set of affordances. Throughout much of human history, children's play has probably been treated in a fairly matter-of-fact sort of way. Much writing on children's play has come from educationists and psychologists, but this has critically focused on play in contemporary western societies, such as the USA or UK.

Piaget $^{[14]}$ was one of the first to describe a developmental sequence in children's play. This went from "practice play", through "symbolic play" (fantasy/pretend play), to "games with rules". Piaget saw these as overlapping stages through the childhood years and they were linked to his cognitive developmental theory. By "practice play", he mainly meant early sensorimotor play in infants (as well as most animal play). "Symbolic play" became possible when the symbolic function was developed-the child was able to represent objects in the world around, internally. From this point, pretend or fantasy play becomes possible-a period Piaget saw as beginning from around 18 months. From around 6 years he saw symbolic play as being superseded by "Games with rules". Here the activity is governed by public rules, which must be followed, usually in co-ordinated play with other players, as in games of soccer, or hopscotch, 
or marbles (which Piaget studied, watching children in Neuchâtel, near Geneva) ${ }^{[15]}$.

Although theoretical and empirical arguments can be marshaled to support either position, research and theory in the area of behavioral genetics would suggest that children from richer quality home environments would benefit most. For the past half century, considerable effort has been devoted to mapping the relations between the home environment and selected aspects of the child's development. Perhaps the most notable attempt in this area is the HOME Inventory by Linver et al. ${ }^{[16]}$ attempted to measure the environmental and interaction factors believed to be associated with adequate child welfare services. The Home Observation for Measurement of the Environment (HOME) Inventory was designed to measure the quality and quantity of stimulation and support available to a child in the home environment. The focus is on the child in the environment and the child as a recipient of inputs from objects, events and transactions occurring in connection with the family surroundings. Although the HOME inventory was not designed to specifically examine the relationship to child motor development, one of the most striking and consistent findings has been' availability of stimulating play materials" as a predictor of future mental behavior ${ }^{[17]}$.

Abbott et al. ${ }^{[18]}$ used three subscales of the HOME inventory (maternal responsivity, provision of appropriate learning materials and maternal involvement) and the Alberta Infant Motor Scale (AIMS) to assess 43 homes and their 8 month-old children's motor development. Results did not show any significant correlation between the HOME and the AIMS scores, but the children in the study scored high both on the AIMS and on the HOME. These results led the authors to conclude that despite the lack of statistically significant support for the hypothesized relation between home environment and motor development, related evidence suggests that more supportative home environment is associated with higher infant motor development. According to them, lack of sensitivity in the HOME inventory (ceiling effect), questionable validity of the HOME inventory to support infant motor development, homogeneity of family aspects (median and high SES) and significantly high motor scores with the AIMS, could have combined to reflect the results. The researchers concluded that although the home environment is surely within the host of subsystems that contribute to infant motor development, little research exists examining this relationship. Furthermore, they strongly emphasized that, "a valid measure reflecting aspects of the home environment that support infant motor development needs to be created". Arguably, such an instrument could have potential for enhancing our understanding of the role of the home on early childhood motor development. In addition, such an instrument could provide useful information in a wide variety of settings, including clinical research with applications to intervention and remediation. For example, medical professionals and social workers could use the instrument to assess the home environment and then provide resources or recommendations to enhance its potential in maximizing development ${ }^{[19]}$.

Goyen and Lui ${ }^{[20]}$ examined motor development at 18, 36 and 60 months in a group of 58 "apparently normal" high-risk infants. Their intent was to determine the relation of motor behavior to the quality of the home environment as measured by the HOME inventory. They concluded that the home environment differently influenced the development of gross and fine motor skills. Infants with a lower HOME score consistently scored poorer on Peabody motor scores; however, the difference was only significant for the gross motor skills.

Bober et al. ${ }^{[21]}$ used 6-month-olds to examine the specific interaction of two features of the home environment, availability of toys and amount of maternal involvement. The researchers found that higher locomotor, eye-hand coordination and critical developmental quotients were associated with the additive combination of more optimal play materials and high level of maternal involvement. When examining the independent contribution of the factors, appropriate play materials were associated with more favorable eye-hand coordination.

Bartlett and Kneale Fanning ${ }^{[22]}$ determined the relationship between both use of infant equipment and play positions and motor development of infants born preterm who were classified as high risk. Subjects were 60 parent-infant dyads attending a developmental follow-up clinic. Parents reported the duration of infant equipment use and the predominant positions in which their infants played in the previous month. Infants were assessed using the Alberta Infant Motor Scale (AIMS). The results showed that Equipment use was not related to motor development; however, the duration of carrying was negatively related to the sit subscale of the AIMS $(r=-0.31, p=-0.05)$. As a group, the infants in this sample spent more time in the relatively less active play positions of sitting and supine than in the positions of prone and standing.

Considering Bartlett and Kneale Fanning ${ }^{[22]}$ study, Therapists should consider the use of equipment and specific play positions to enhance motor development of infants born preterm and work with parents to 
promote an understanding of the importance of providing their infants with opportunities to develop early motor competencies.

Rodrigues $^{[23]}$ was the first which developed a new inventory for Assessing Affordances in the Home Environment for Motor Development (AHEMD-SR). His research addresses gap with the goal of creating an innovative parental self-report instrument for assessing the quality and quantity of factors (affordances and events) in the home that are conducive to enhancing motor development in children ages 18-42 months. Validity and reliability was examined in two studies, using Portuguese and US families. He hypothesized that affordances are organized according to a common structure that can be represented by a number of specific stable dimensions of the home environment. Study 1, following initial face validity determination, expert opinion feedback and selective pilot-testing, construct validity was examined using 381 Portuguese families. Reliability was established through the scale reliability coefficient with a value of 0.87 . Study 2 tests for the content validity of the instrument, examining the relationship between the inventory and level of motor development. Fifty-one participants from the original sample were assessed for motor development using the Peabody Developmental Motor Scales II (PDMS2). Comparisons were made between the PDMS2 classifications of the AHEMD-SR quartile groups. Results supported the primary hypothesis, that is, less favorable motor development was associated with less availability of home affordances. Furthermore, the interaction of (factors) Inside Space and Variety of Stimulation was significantly related to both Gross and Total Motor Development scores. The findings of these two studies suggest that the AHEMD-SR is a valid and reliable instrument for assessing how well home environments afford movement and potentially promote motor development.

Although specific home environment and motor development characteristics have been examined the fact remains that minimal information is available in relation to the multidimensional effects of the home, especially on motor development. Considering that, there is no research on the subject has been done in Iran. The main objective of this investigation is to analyze the validity and reliability of a new Inventory for Assessing Affordances in the Home Environment for Motor Development (AHEMD-SR). This will help to assess the quality and quantity of motor development opportunities in the home during early childhood and to understand how some of activities are related to early motor development. The main research questions are whether AHEMD-SR has high validation and reliability in Iran and whether affordances in the home environment can effect whether affordances in the home environment can effect motor development of children age 18-42 month. To answer these questions, the following hypotheses (primary and secondary) are proposed:

Hypotheses 1: AHEMD-SR is a valid and reliable instrument for assessing how well environments home afford movement and potentially promotes motor development of children age 18-42 months.

Hypotheses 2: There is a positive relationship between outside physical space, inside physical space, variety of stimulation, gross motor materials and fine motor materials (five factors of AHEMD-SR) and level of motor development in children age 18-42 months. It was hypothesized that a high AHEMD score complements the likelihood of a high motor development score.

Hypotheses 3: There is a multiple relationship between outside physical space, inside physical space, variety of stimulation, gross motor materials and fine motor materials (five factors of AHEMD-SR) and level of motor development in children age 18-42 months.

Hypotheses 4: Fine motor toys is a predictor for level of child motor development.

In the present study the term Affordances defined as opportunities that offer the individual potential for action and consequently to learn and develop a skill or a part of the biological system ${ }^{[11-13]}$.

Affordances are related to motor skills. Motor skills are skills that child gain throughout early motor developments. There are two types of motor skills: Gross motor skills and fine motor skills which are important terms in a child's development.

Gross motor skills are movements of the large muscles in the body; such as arm and leg muscles. These types of movements are easier for a child to control and usually develop faster then fine motor skills. Some of the movements that are considered gross motor are running, walking, skipping, climbing, crawling, rolling over and dancing to name just a few. Fine motor skills are movements of the small muscles in the body such as hands. These are the hardest for a child to develop because in order to have fine motor skills you have to have really good control over your body. Young children do not tend to have as many fine 
motor skills as gross motor. Some of the movements that are considered fine motor are writing, pointing, grasping, holding and reaching ${ }^{[3]}$.

\section{MATERIALS AND METHODS}

Babies are born with a sophisticated brain that is pre-programmed to learn and react to its environment. Development in the first three years of life is incredibly quick. Before children can move about on their own, they have to learn to control their movements. Walking and talking are probably amongst the most eagerly awaited moments for parents. However, before children can walk, they have to be able to control their movements. These early beginnings of motor skills mark a major milestone in development. A contemporary view of early childhood motor development considers environmental influences as critical factors in optimal growth and behavior, with the home being the primary agent.

Rodrigues $^{[23]}$ developed self-reporting research instrument that assesses the quality and quantity of motor development affordances in the home for children 18-42 months-the AHEMD-SR. This Inventory consists of 67 questions addressing home and family characteristics. That is, questions were structured in an unbiased and affirmative (positive) manner. For example, "My child plays with other children as a usual and ordinary daily event". As opposed to "My child usually doesn't play with other children as a daily event". Readability was set at an approximate fourth grade reading level. This Inventory comprised five sections. Two sections comprising on Child and Family Characteristics and three on home environment characteristics and affordances: Physical Space, Daily Activities and Play Materials. Three types of questions: simple dichotomic choice, 4-point Likert-type scale and description-based queries were used. For scoring method, Excel calculator program was designed spatially for this inventory. Reliability was established through the scale reliability coefficient with a value of 0.87 with a standard error of 0.023 and a $95 \%$ respective confidence intervals ranging from 0.82-0.91, which indicated a high consistency of the instrument for measuring the construct of interest.

The research population is the families with children of age 18-42 months in Ahvaz city of Iran. A sample of 350 families was selected for motor assessment. Children were chosen according to the criteria of age (18-42 months) and absence of disabilities from Child Care Centers. In an effort to obtain similar socioeconomic status, city divided into four areas. From each area, two Child Care Centers were elected through random selection. From this initial pool, fifteen families did not return the inventory and permission to perform motor testing was denied by the parents of four children. Thus the final sample consisted of 328 participants (145 girls, 183 boys) with mean age of 35.0 \pm 5.2 months that completed AHEMDSR.

Directors of the eight early childhood centers sent each child's family a letter explaining the purpose of the study, asking for their collaboration and providing consent forms. Testing days were scheduled in accordance with the center director. The AHEMD-SR was completed by the child's family. A coded number was assigned to each family/child, in order to keep the researchers naive to the results until all testing procedures were completed. It took families two weeks to complete and hand the inventory back to childhood centers. As noted before some families did not return the inventories. According to that the sample was decreased to 328 families.

The scoring method was done, using Excel calculator program which designed spatially for this inventory. According to the results, total scores ranged from 0-20. Total AHEMD classification under 10 was described as low, which means house provides few opportunities for child's motor development. Total AHEMD classification 10-15 was described as Average, which means house provides sufficient opportunities for child's motor development. And Total AHEMD classification 15-20 was described as High, which means house provides very good opportunities for child's motor development.

The collected scores were imported in to Scientific Package of Social Sciences (SPSS) software. The data were analyzed using correlation statistics. In this study Internal consistency reliability using Cronbach's alpha, was 0/88 indicating high level of internal consistency.

In this study, formal validity (expert opinion feedback) and construct validity (relations between the inventory results and level of motor development) was examined. According to absence of a valid inventory for measuring child motor development in Iran, for Content validity of AHEMD-SR, five critical questions was made from five factors (Outside Physical Space, Inside Physical Space, Variety of Stimulation, Gross Motor Materials and Fine Motor Materials) of AHEMD-SR. So participants from the original sample were assessed for motor development using five critical questions on 5 point Likert-type scale:

- How do you consider the outside space of your house for your child motor development? 
- How do you consider the inside space of your house for your child motor development?

- How do you consider the daily activities in your house for your child motor development?

- How do you consider your child's play materials used for gross movements with arm and leg (such as running, walking, skipping, climbing and crawling)?

- How do you consider your child's play materials used for fine movements with hands (such as writing, pointing, grasping, holding and reaching)?

Comparisons were made between critical questions classifications of the AHEMD-SR and factors of AHEMD-SR.

\section{RESULTS}

Prior to testing the study's hypotheses, descriptive statistics were computed (mean and standard deviation and range) to assure that inferential assumptions were met. These are shown in Table 1.

According to Table 1 a higher mean value was found for Fine motor Toys. Total AHEMD scores were calculated adding each subscale classification. Ranging from 6-20, this total score presented a normal distribution with an average of 12.5 and a SD of 3.179. As mentioned before AHEMD classification 10-15 was described as Average, which means house provides sufficient opportunities for child's motor development.

The first main research question is whether AHEMD-SR has high validation and reliability in Iran. Formal validity and construct validity were used to answer this question. Formal validity of the instrument established by expert opinion feedback witch showed very high formal validity of 0.92 . Construct validity of the instrument established by relations between the inventory results and five critical questions which had been made to characterize level of motor development as shown in Table 2.

According to Table 2, Construct validity was 0.75 which supported the primary hypothesis, that is, AHEMD-SR is a valid and reliable instrument for assessing how well environments home afford movement and potentially promotes motor development of children age 18-42 months.

Table 1: Descriptive statistics for the variables in this study

\begin{tabular}{llll}
\hline Variable & Range & Mean & SD \\
\hline Outside space & $1-4$ & 2.185 & 1.750 \\
Inside space & $1-4$ & 3.333 & 1.177 \\
Variety of stimulation & $1-4$ & 3.000 & 0.919 \\
Fine motor toys & $1-4$ & 1.519 & 0.893 \\
Gross motor toys & $1-4$ & 1.593 & 0.797 \\
Total AHEMD & $6-20$ & 12.55 & 3.179 \\
\hline
\end{tabular}

The second main research question is whether affordances in the home environment can effect motor development of children age 18-42 months. Pearson Product-Moment Correlations (PPMC) and Univariate ANOVAs were computed to answer this question.

Simple correlations between five factors of AHEMD and total AHEMD are shown in Table 3.

Results indicated a significant $(0.01$ or 0.05$)$ positive moderate correlation between the five factors of AHEMD and total AHEMD. The correlations magnitude ranged between $0.50-0.78$ for Total AHEMD. Fin motor toys correlated strongest with total AHEMD. Total AHEMD was used as dependent variable to compare with five factors (Outside space, inside space, variety of stimulation, gross motor toys and fine motor toys). These results supported the secondary hypothesis, that is, there is a positive relationship between five factors of AHEMD-SR and level of motor development in children age 18-42 months.

Univariate ANOVAs were performed to test for critical effect of subscale classification with dependent variable. As showed in Table 4, regression analyses were implemented to test for the possible linear association between the five AHEMD subscales and their interaction with Total AHEMD scores.

Table 2: Correlations between AHEMD factors and five critical questions. Correlation between total AHEMD and total critical questions

\begin{tabular}{lllllll}
\hline & Q1 & Q2 & Q3 & Q4 & Q5 & QT \\
\hline Outside space & 0.97 & & & & & \\
Inside space & & 0.96 & & & & \\
Variety of stimulation & & & 0.55 & & & \\
Fine motor toys & & & & 0.94 & & \\
Gross motor toys & & & & & 0.81 & \\
Total AHEMD & & & & & & 0.75 \\
\hline
\end{tabular}

$\mathrm{p}<0.01$

Table 3: Correlations between each AHEMD factors and total AHEMD

\begin{tabular}{ll}
\hline Variable & Total AHEMD \\
\hline Outside space & $0.71^{* *}$ \\
Inside space & $0.65^{* *}$ \\
Variety of stimulation & $0.50^{*}$ \\
Fine motor toys & $0.78^{* *}$ \\
Gross motor toys & $0.62 * *$ \\
\hline$*: \mathrm{p}<0.05 ; * * \mathrm{p}<0.01$ &
\end{tabular}

Table 4: Stepwise regression analyses to test for a predictor of total AHEMD

Standardized coefficient

\begin{tabular}{lllll} 
Model & Beta & $\mathrm{R}^{2}$ & $\mathrm{~F}$ & Sig. \\
\hline
\end{tabular}

\begin{tabular}{lllll}
\hline Fine motor toys & 0.800 & 0.640 & 44.45 & 0.000
\end{tabular}

Predictors: (Constant): Fine motor toys; Dependent variable: Total AHEMD 
Table 5: Enter regression analyses to test for the possible linear association between the five AHEMD subscales and their interaction with total AHEMD

\begin{tabular}{lllll}
\hline Model & $\begin{array}{l}\text { Standardized coefficient } \\
\text { Beta }\end{array}$ & $\mathrm{R}^{2}$ & $\mathrm{~F}$ & Sig. \\
\hline 1 & 0.997 & 0.993 & 628.52 & 0.000 \\
\hline
\end{tabular}

Predictors: (Constant): Fine motor toys, outside space, inside space, variety of stimulation andgross motor toys. Dependent variable: Total AHEMD

For regression analysis two techniques were used: Stepwise and Enter. As showed in Table 4, stepwise regression technique showed that fine motor toys ( standardized coefficient $=0.800 ; \mathrm{p}=0.000$ ) are a predictor factor for total AHEMD. This result supported the forth hypothesis and Fine motor toys are a predictor factor for child motor development. Enter regression technique indicated that there is a high multiple relationship between the five factors of AHEMD and Total AHEMD (standardized coefficient $=0.997 ; \mathrm{p}=0.000)$ which supported the third hypothesis (Table 5).

\section{DISCUSSION}

From the results noted, the AHEMD-SR proved its merit in the potential to evaluate and discriminate among different home profiles according to their theoretical driven characteristics for motor development. These data revealed a common structured organization of potential affordances in the home environment comprising five latent factors: Outside space, inside space, variety of stimulation, fine motor toys and gross motor toys. Each of these factors represented a meaningful structure inside the home, possibly resulting from the underlying decisions on how families provide specific environmental stimuli to their children. Although correlation values between factors could imply an overall degree of stability within each home. The relevancy for investigating these different profiles of affordances lies in the potential for each child to improve their motor skills. The findings of this study revealed that the AHEMD-SR is a valid indicator of affordances found in the home environment that have the 'potential' to influence the motor development of young children. As such, this instrument has promise in addressing the statement by Abbott et al. ${ }^{[18]}$ recommending that "a valid measure reflecting aspects of the home environment that support infant motor development needs to be created".

The initial prediction was that with a low AHEMD score is the likelihood of a lower motor development score. Accordingly, selection of the sample was aimed to collect a range of environmental characteristics (home location, socioeconomic status and family structure) and motor development behaviors. There was a positive correlation between five factors of AHEMD and Total AHEMD that is prove for importance of affordances of home in child motor development. High score in each factor is mid with high AHEMD score which means there are good opportunities for child's motor development.

According to validity and reliability of AHEMD$\mathrm{SR}$, it is a very good instrument for assessing the quality and quantity of motor development affordances in the home for children 18-42 months.

Final results from regression showed that there is there is a high multiple relationship between the five factors of AHEMD and Total AHEMD and Fine Motor Toys is a predictor for Total AHEMD score. As mentioned before Fine motor development is important part of child's development. Fine motor skills are movements of the small muscles in the body such as hands. These are the hardest for a child to develop because in order to have fine motor skills you have to have really good control over your body. Young children do not tend to have as many fine motor skills as gross motor. Fine motor skills can be very hard for children sometimes because they require a lot of control over their bodies. Children develop fine motor skills by writing, drawing, painting, putting puzzles together, etc. As children get older their toys get smaller and that is because they can manipulate things easier. Puzzles for infants usually have handles on them, then the handles disappear and it is only 5 or 6 puzzle pieces and as a child gets older pieces get smaller and have more intricate shapes ${ }^{[25]}$. Because fine Motor movements are harder for a child to control and usually develop slower then gross motor skills improvements of fine motor skills means child motor developments has been improved. So it can be a significant predictor for motor Total AHEMD score and child motor development.

Of most importance to this study is the insight obtained from the Total AHEMD scores because they take into account not only the number but also the variety of affordances. Regression analyses revealed that the Fine Motor Toys was a significant predictor for Total AHEMD scores. This finding suggests that a proper amount of fine movements in the house can multiply the effect of stimulation and spaces. Overall, these results show promising evidence for supporting the prediction that with a low AHEMD score is the likelihood of a lower motor development score. Less favorable motor development was associated with less availability of home affordances and the interaction of inside space and gross motor toys was significantly related to both Fine and Total motor development. Additional research needs 
to include samples involving a wider variation of motor development, especially in the lower range.

\section{CONCLUSION}

For the past 40 years, effort has been devoted to mapping the relations between the home environment and selected aspects of the child's development. Although motor items have been included in noted inventories, such as the $\mathrm{HOME}^{[24]}$, the fact remains that minimal information is available in relation to the multidimensional effects of the home on motor development. The starting premise of this study, founded in selected propositions of Ecological (Affordance) theory ${ }^{[9,10]}$ was that the home environment can provide affordances that can be conducive to stimulating motor development. It was further hypothesized that affordances are organized according to a common structure that can be represented by a number of specific stable dimensions of the home environment. After developing AHEMD$\mathrm{SR}^{[23]}$, a valid measure reflecting aspects of the home environment that influence early motor development, there is still lack of researches using AHEMD-SR in different cultures.

A common structure was found that represents the characteristics present in the home environment that can be conducive to motor development. This structure, represented by five latent dimensions (outside physical space, inside physical space, variety of stimulation, gross motor materials and fine motor materials) can be reliably, advantageously and parsimoniously assessed by the parent's answers to the AHEMD-SR. Furthermore, the AHEMD-SR revealed potential to evaluate and discriminate among different home profiles according to their theoretically driven characteristics for motor development. Each of the dimensions represented a meaningful structure associated with the home, possibly resulting from the underlying decisions on how families provide specific environmental stimuli to their children. The better fit of the 5-factor model and the existence of key significant correlation values, suggests that parent's decisions were not (or could not) always be consistent across dimensions. This assumption complements the notion of individual differences in children that are likely between and within homes. Less favorable motor development was associated with less availability of home affordances and Total motor development.

Although the AHEMD-SR was initially developed as a research instrument to enhance our basic understanding of the potential of the home environment in optimizing motor development of the child, its use in clinical and educational settings has equally significant potential. For example:

- The AHEMD-SR can be used to improve the relationship between teacher and parent and therefore increase the level of child readiness for learning

- This instrument can be used as a tool for early intervention. That is, assessment of the home (by parent, professional, or future teacher) followed up with recommendations for home modification and parental education by the early childhood professional. As noted earlier, developmentally appropriate plays materials and parental stimulation can be strong influences in child development. This observation may be especially relevant to children at risk. In regard to possible expansion of this study, additional research needs to include greater sample sizes, involving a wider variation of motor development, especially in the lower range. Complementing this fact is also the need for expanding the age range of the instrument. Given the trend toward early intervention, an AHEMDSR for ages 3-18 months and perhaps one for 42-72 months (entering the school years) is warranted

- Comparing AHEMD scores with later academic performance. This could be a major complement to the findings of the HOME Inventory mentioned earlier. Possible avenues of inquiry could include a study of the relationship between early experience with specific toys and play materials and (for example) handwriting ability and cognitive development in preschool and primary grades

- Observing the longitudinal characteristics of the instrument. For example, tracking AHEMD-SR scores and behavior (motor and mental) over time. Testing the instrument's clinical significance for early intervention. For example, as a follow-up to assessing the home, the environment could be modified to include developmentally appropriate fine-motor materials-then, over time, examine the effects on behavior. This may be especially interesting with high-risk populations such as infants born with low-birth weight, or from lowincome homes

- Given that level of stimulation is a potentially significant factor, future research should consider a more in-depth analysis of this component. Currently, the questions related to level of stimulation are somewhat broad; these could be expanded and modified for greater detail depending on the specific research questions addressed

- Examining cross-cultural characteristics; comparing home environments from different 
cultures. The anticipated contributions are found in the instrument's research and clinical applications

Another appropriate question that needs to be addressed is the instrument's stability over time. For example, does change in the home overtime complement change in motor behavior? And, as noted earlier, an avenue of research that is of interest to many early childhood educators is a study of the interrelationships between home affordances that stimulate motor development and later academic performance. In light of the long-term goal of increasing the cultural scope of the instrument, for further validation in different settings and populations are also in order. There is little doubt that there are differences in infant behavior among cultural groups around the world and subgroups within a country. It would be interesting to determine, for example, which factors and items from the AHEMD-SR remain stable across cultures. Common variables in investigations of this type include relationship to parental expectations, Socioeconomic Status (SES), child rearing practices, parent education and space. In this dissertation, although a careful selection was made of a Iranian sample that was comparable in SES and parent education, living space and child-rearing differences were probable. However, as one would expect, within any cultural sample is wide range of variability in those factors.

In summary, the findings of this study suggest that the AHEMD-SR is a valid and reliable instrument for assessing how well home environments afford movement and potentially promote motor development. However, although it can make reasonable predictions about developmental outcome, one should keep in mind that the margin of error can vary considerably. The interaction of nature and nurture results in individuality that stresses our ability to measure with a high degree of accuracy-the human condition. Our expectation is that the AHEMD-SR would be a step further in the right direction to open new avenues into understanding the multifaceted dynamics and interaction of the home environment and motor behavior. The present results proved optimistic on this matter, but more data is paramount to better isolate the motor affordance effect within the complex ecology of children's live.

\section{REFERENCES}

1. Bronfenbrenner, U., 2000. Ecological Theory. In: Encyclopedia of Psychology, Kazdin, A.E. (Ed.). American Psychological Association and Oxford University Press, Washington DC., USA., ISBN: 155798-187-6, pp: 129-133.
2. Diamond, A., 2000. Close interrelation of motor development and cognitive development of the cerebellum and prefrontal cortex. Child Dev., 71: 44-56. DOI: 10.1111/1467-8624.00117

3. Kurtz, L.A., 2008. Understanding Motor Skills in Children with Dyspraxia, ADHD, Autism and other Learning Disabilities: A Guide to Improving Coordination. Jessica Kingsley Publishers, London, ISBN: 1843108267, pp: 160.

4. Shapiro, B.K. and H. Gwynn, 2008. Neurodevelopmental Assessment of Infants and Young Children. In: Developmental Disabilities in Infancy and Childhood, Accardo, P.J. and J.A. Accardo Washington (Eds.), Vol. 1, Paul H. Brooks, Philadelphia, ISBN: 9781557667588, pp: 311-322.

5. Allen, M.C. and G.R. Alexander, 1997. Using motor milestones as a multistep process to screen preterm infants for cerebral palsy. Dev. Med. Child Neurol., 39: 12-16. DOI: $0.1111 / j .1469-$ 8749.1997.tb08198.x

6. Thelen, E., 1995. Motor development: A new synthesis. Am Psychol., 50: 79-95. http://www.ncbi.nlm.nih.gov/pubmed/7879990

7. Bronfenbrenner, U. and S.J. Ceci, 1994. Naturenurture reconceptualized in developmental perspective: A bioecological model. Psychol. Rev., 101: 568-586.

http://direct.bl.uk/bld/PlaceOrder.do?UIN=021369

093\&ETOC $=$ EN\&from $=$ searchengine

8. Bronfenbrenner, U., 1981. The Ecology of Human Development: Experiments by Nature and Design. Harvard University, Cambridge, MA., ISBN: 10: 0674224574, pp: 348.

9. Gibson, J.J., 1986. An Ecological Approach to Perception. Lawrence Erlbaum Associates, Inc., Boston, MA., ISBN: 0898599598, pp: 332.

10. Gibson, E.J., 2001. Perceiving the Affordances: A Portrait of Two Psychologists. 1st Edn., Lawrence Erlbaum, Mahwah, New Jersey, USA., ISBN: 0805839496, pp: 160.

11. Heft, H., 1997. The Relevance of Gibson's Ecological Approach to Perception for Environment-Behavior Studies. In: Advances in Environment, Behavior and Design: Toward the Integration of Theory, Methods, Research and Utilization, Moore, G.T. and R.W. Robert (Eds.), Vol. 4, 1st Edn., Springer, New York, ISBN: 10: 0306453436, pp: 71-108.

12. Stoffregen, T.A., 2000. Affordances and events: Theory and research. Ecol. Psychol., 12: 93-107. http://www.informaworld.com/smpp/ftinterface?co ntent $=\mathrm{a} 784403179 \& \mathrm{rt}=0 \&$ format $=\mathrm{pdf}$ 
13. Hirose, N., 2002. An ecological approach to embodiment and cognition. Cognit. Syst. Res., 3: 289-299. DOI: $10.1016 /$ S13890417(02)00044-X

14. Piaget, J., 1962. Play, Dreams and Imitation in Childhood. WW Norton and Co Inc., London, ISBN: 10: 0393001717, pp: 308.

15. Smith, P.K., 2009. Children and Play: Understanding Children's Worlds. John Wiley and Sons, Hoboken, New Jersey, USA., ISBN: 978-0631-23521-7, pp: 272.

16. Linver, M.R., J. Brooks-Gunn and N. Cabrera, 2004. The Home Observation for Measurement of the Environment (HOME) inventory: The derivation of conceptually designed subscales. Parent Sci. Pract., 4: 99-114. http://www.informaworld.com/smpp/1418815670$6132724 /$ content $\sim \mathrm{db}=\mathrm{all} \sim$ content $=\mathrm{a} 785828584$

17. Bradley, R.H., B.M. Caldwell and R.F. Corwyn, 2003. The child care HOME inventories: Assessing the quality of family child care homes. Early Childhood Res. Q., 18: 294-309. DOI: 10.1016/S0885-2006(03)00041-3

18. Abbott, A.L., D.J. Bartlett, J.E.K. Fanning and J. Kramer, 2000. Infant motor development and aspects of the home environment. Pediatr. Phys. Ther., 12: 62-67. http://direct.bl.uk/bld/PlaceOrder.do?UIN=079844 $184 \&$ ETOC $=$ RN\&from $=$ searchengine

19. Mundfrom, D.J., R.H. Bradley and L. Whiteside, 1993. A factor analytic study of the infant-toddler and early childhood versions of the HOME inventory. Educ. Psychol. Measure., 53: 479-489. DOI: $10.1177 / 0013164493053002017$

20. Goyen, T.A. and K. Lui, 2002. Longitudinal motor development of "apparently normal" high-risk infants at 18 months, 4 and 5 years. Early Hum. Dev., 70: 103-115. DOI: 10.1016/S03783782(02)00094-4
21. Bober, S.J., R. Humphry, H.W. Carswell and A.J. Core, 2001. Toddlers' persistence in the emerging occupations of functional play and selffeeding. Am. J. Occup. Ther., 55: 369-376. http://www.ncbi.nlm.nih.gov/pubmed/11723980

22. Bartlett, D.J. and J.E. Kneale Fanning, 2003. Relationships of equipment use and play positions to motor development at eight months corrected age of infants born preterm. Pediatr. Phys. Ther., 15: 8-15.

DOI: 10.1097/01.PEP.0000051693.49199.41

23. Rodrigues Luis Paulo, Saraiva Linda and Gabbard Carl, 2005. Development and construct validation of an inventory for assessing the home environment for motor development. Res. Q. Exerc. $\quad$ Sport, $\quad$ 140 $\quad 148$. http://cat.inist.fr/?aModele $=$ afficheN\&cpsidt $=1696$ 4518

24. Han, W.J., T. Leventhal and M.R. Linver, 2004. The Home Observation for Measurement of the Environment (HOME) in middle childhood: A study of three large-scale data sets. Parent. Sci. Pract., 4: 189-210. DOI: 10.1207/s15327922par0402\&3_5

25. Cohen, D., 2006. The Development of Play. 3rd Edn., Routledge Publisher, New York, ISBN: 0415347017, pp: 206. 